\title{
Prognostic Influence of Tobacco Smoking on Human Papilloma Virus-Related Oropharyngeal Cancer is Dependent Upon Treatment Modality
}

Jun ITAMI ( $\nabla$ jitami@ncc.go.jp )

National Cancer Center Hospital: Kokuritsu Gan Kenkyu Center Chuo Byoin https://orcid.org/00000003-1579-7406

\section{Kenya KOBAYASHI}

National Cancer Center Hospital: Kokuritsu Gan Kenkyu Center Chuo Byoin

\section{Taisuke MORI}

National Cancer Center Hospital: Kokuritsu Gan Kenkyu Center Chuo Byoin

\section{Yoshitaka HONMA}

National Cancer Center Hospital: Kokuritsu Gan Kenkyu Center Chuo Byoin

\section{Yuko KUBO}

National Cancer Center Hospital: Kokuritsu Gan Kenkyu Center Chuo Byoin

Naoya MURAKAMI

National Cancer Center Hospital: Kokuritsu Gan Kenkyu Center Chuo Byoin

Go OMURA

National Cancer Center Hospital: Kokuritsu Gan Kenkyu Center Chuo Byoin Yuko NAKAYAMA

National Cancer Center Hospital: Kokuritsu Gan Kenkyu Center Chuo Byoin Hiroshi IGAKI

National Cancer Center Hospital: Kokuritsu Gan Kenkyu Center Chuo Byoin Kae OKUMA

National Cancer Center Hospital: Kokuritsu Gan Kenkyu Center Chuo Byoin Koji INABA

National Cancer Center Hospital: Kokuritsu Gan Kenkyu Center Chuo Byoin

Kana TAKAHASHI

National Cancer Center Hospital: Kokuritsu Gan Kenkyu Center Chuo Byoin

\section{Tairo KASHIHARA}

National Cancer Center Hospital: Kokuritsu Gan Kenkyu Center Chuo Byoin

\section{Tomoya KANEDA}

National Cancer Center Hospital: Kokuritsu Gan Kenkyu Center Chuo Byoin

\section{Satoshi SHIMA}

National Cancer Center Hospital: Kokuritsu Gan Kenkyu Center Chuo Byoin 


\section{Ayaka TAKAHASHI}

National Cancer Center Hospital: Kokuritsu Gan Kenkyu Center Chuo Byoin

\section{Yuri SHIMIZU}

National Cancer Center Hospital: Kokuritsu Gan Kenkyu Center Chuo Byoin

\section{Seiichi YOSHIMOTO}

National Cancer Center Hospital: Kokuritsu Gan Kenkyu Center Chuo Byoin

\section{Research}

Keywords: oropharyngeal squamous cell carcinoma, HPV-related, p16, tobacco smoking, prognosis

Posted Date: October 1st, 2020

DOI: https://doi.org/10.21203/rs.3.rs-83235/v1

License: (c) (1) This work is licensed under a Creative Commons Attribution 4.0 International License.

Read Full License 


\section{Abstract}

[Purpose] Tobacco smoking has been reported to influence the prognosis of human papilloma virus (HPV)-related orophageyngeal squamous cell carcinoma (OPSCC). However, it remains to be studied whether tobacco smoking equally affects the patients treated by various modalities.

[Material and Method] From 2010 through 2018, 241 patients with OPSCC were treated in a single institution, out of which 144 patients had HPV-related OPSCC. P16 immunohistochemical staining was used as a surrogate of HPV infection. Two patients was excluded because of inadequate radiation dose, and the remaining 142 patients were the subject of this study. Median age was 63.8 years and more than $80 \%$ were male. More than $70 \%$ were smokers or ex-smokers with a median pack year of 17.3 . Eighty-seven patients (61.3\%) were classified as stage I.

[Results] For all 142 patients with HPV-related OPSCC, overall survival (OS) and disease-specific survival (DSS) were $87.0 \%$ and $93.4 \%$ in 3 years, respectively. There were no differences of OS and DSS according to the stages by $8^{\text {th }}$ edition of tumor, node, and metastasis (TNM) classification and the primary sites. OS and DSS were different by the amount of tobacco smoking expressed in pack year (PY) $\geq 30$ and $<30$. Also the presence of secondary cancer impacted OS. However, the influence of the amount of tobacco smoking was reduced in the patients treated by radiation therapy.

[Conclusions] The impact of tobacco smoking upon the prognosis of HPV-related OPSCC seems to be dependent upon therapeutic modalities.

\section{Introduction}

Human papilloma virus (HPV)-related oropharyngeal squamous cell cancer (OPSCC) is increasing in frequency in the developed countries and it has been demonstrated that HPV-related OPSCC has a favorable prognosis in comparison to HPV-unrelated $\operatorname{OPSCC}(1,2)$. Although one of the major etiological factors of HP-unrelated OPSCC is tobacco smoking, it plays only a minor role in the etiology of HPVrelated OPSCC. In contrast, it is repeatedly shown that tobacco smoking influences prognosis of HPVrelated and HPV-unrelated OPSCCs similarly $(3,4)$. However, whether tobacco smoking is equally affecting the patients treated by various modalities is quite open for discussion.

In this single institutional retrospective study, all the patients with HPV-related OPSCC treated from 2010 through 2018 were studied, irrespective of the treatment modalities, and the influence of tobacco smoking on variously treated patients was analyzed in the HPV-related OPSCC.

\section{Material And Method}

From 2010 through 2018, 241 patients with non-metastatic OPSCC of a known p16 status were treated in a single institution, of which 144 patients had p16-positive OPSCC and 97 had p16-negative OPSCC. For the demonstration of HPV infection of the tumor cells, p16 immunohistochemical staining was used as a 
surrogate in this study $(5,6)$. An expert head and neck pathologist (YM) exclusively diagnosed p16 immunohistochemical examination as positive if cytoplasmic as well as nuclear stainings were obvious in more than $75 \%$ of tumor cells. Two patients out of these 144 with p16-positive HPV-related OPSCC were treated palliatively with radiation dose less than $50 \mathrm{~Gy}$ and excluded from this study and the remaining 142 patients treated with a curative intent are the subject of this study. Clinical characteristics of the 142 patients are shown in Table 1. Median age of the patients was 63.8 years with a range from 35 to 84 years. More than $80 \%$ of the patients were male. One-hundred one patients $(71.1 \%)$ were current smokers or former smokers. The remaining 41 patients $(28.5 \%)$ were never-smokers. The amount of tobacco smoking was expressed by pack year (PY) with a median of 17.3. Time interval between smoking cessation and treatment initiation of OPSCC ranged from 0 day to 50 years with a median of 9.48 years. Synchronous or metachronous secondary cancers were found in 45 patients (31.7\%).

Staging procedures were performed with physical examinations, endoscopy, computed tomography, and magnetic resonance imaging (MRI). Positron emission tomography (PET)-CT or PET-MRI was performed in selected patients. More than $70 \%$ of the patients had a primary lesion in the lateral wall of oropharynx, which was followed by the anterior wall (base of tongue) primary in frequency. Stage classification was done according to 8 th edition TNM classification (TNM-8). About $60 \%$ of all were classified into stage I.

As for treatment, 81 (56.3\%) of all underwent a definitive operative resection with/without adjuvant radiation and chemotherapy. In 13 of the 81 patients, neck dissection was not performed and only the primary lesions were resected. Radiation therapy with/without chemotherapy were delivered to the remaining 61 patients. Intensity modulated radiation therapy (IMRT) was employed in 98 out of 100 irradiated patients. Clinical target volume was treated up to 50-76 Gy in a conventional fractionation with a mean of $67.8 \mathrm{~Gy}$. Less than $60 \mathrm{~Gy}$ was applied only in 2 patients who were irradiated postoperatively. In 57 patients, chemotherapy with cis-diamine-dichloro-platinum (CDDP) was delivered concurrently with radiation therapy. In 9 patients, cetuximab simultaneously with radiation therapy was administered because of the renal dysfunction. No patients were treated by chemotherapy alone.

Overall survival (OS) and disease-specific survival (DSS) were calculated by Kaplan-Meier method assuming the date of treatment initiation as day 0 . For calculation of DSS, death by the tumor was considered as an event and death without recurrence as censored. Difference between survival curves was tested by log-rank. Chai-square test was used to analyze difference in the incidence of categorical variables. Multivariate analysis using Cox proportional hazard regression models was performed with OS and DSS as an endpoint using PY and the presence of secondary cancer as covariates. All analyses were conducted with SPSS ver. 26. Median follow-up was 44 months.

This single institutional retrospective study was approved by the Institutional Review Board (No. 2017091 and $2018-179)$.

\section{Results}


For all 142 patients with HPV-related OPSCC, OS and DSS were $87.0 \%$ and $93.4 \%$ in 3 years, respectively. By clinical stage, 3 -year OS was $88.5 \%$ in stage I, $84.2 \%$ in stage II, and $83.7 \%$ in Stage III (Fig. 1). There could not be seen any statistically significant differences in OSs by stages. Three-year DSS was $96.1 \%$ in stage I, $88.9 \%$ in stage II, and $87.7 \%$ in Stage III, respectively (Fig. 1), without statistically significant differences. Statistically significant differences in OS and DSS were not reached by various treatment modalities as well as by the primary sites.

In contrast, there were statistically significant differences in OS and DSS by the amount of tobacco smoking. In the patients with PY $\geq 30$, 3-year OS was $74.3 \%$ and 3-year DSS was $84.1 \%$ (Fig. 2). In the patients with PY $<30$, 3-year OS and DSS were $92.9 \%$ and $97.6 \%$, respectively (Fig. 2). Between the patients with $P Y \geq 30$ and $<30$, there were statistically significant differences in OS $(p=0.003)$ and DSS $(p=0.019)$. Time from cessation of tobacco smoking and treatment initiation of OPSCC exerted no significant influences upon OS and DSS.

OS was also influenced by the presence of secondary cancer. Three-year OS was $94.5 \%$ for the patients without secondary cancer, while $73.6 \%$ for the patients with secondary cancer $(p=0.003)$. OS diverged after 2 years by the presence of secondary cancer. In contrast, DSS was not different between the patients with and without secondary cancer $(p=0.432)$.

To see in detail whether patient characteristics and treatment strategy were different by the amount of tobacco smoking, patient characteristics and treatment were classified by PY $\geq 30$ and $P Y<30$ (Table 1). Only the presence of secondary cancer $(p=0.006)$ showed statistically significant differences by chisquare test, with secondary cancers seen more frequently in the patients with $P Y \geq 30$ (Table 1).

To elucidate the impacts of tobacco smoking on prognosis of the patients managed by various modalities, the patients managed with radiation, operation, and chemotherapy were analyzed separately. Figure 3 disclosed that the OSs were different with a nearly statistical significances or with a statistically significance between the patients with $P Y \geq 30$ and $<30$, irrespective of the treatment. In contrary, DSS was not different with a statistical significance between $P Y \geq 30$ and $P Y<30$ in the patients managed with a treatment including radiation therapy $(p=0.254)$. In the patients to whom chemotherapy was delivered, DSS was different with a statistical significance $(p=0.022)$ between $P Y \geq 30$ and $P Y<30$.

By using PY and the presence of secondary cancer as categorical covariates, four multivariate analyses were performed in all the 142 patients, in the patients treated by radiation therapy, in the patients treated by surgery, and in the patients treated with chemotherapy. In the patients treated with radiation therapy and with chemotherapy, the amount of PY lost a statistical significance in DSS, while the difference of DSS by PY remains statistically significant in the whole 142 patients and in the patients who underwent surgery (Table 2). In DSS, presence of secondary cancer was not a significant prognostic factor.

\section{Discussion}


HPV-related OPSCC is known for its different etiology and therapeutic response from HPV-unrelated OPSCC $(1,2)$. HPV-related OPSCC is caused by infection of the high risk HPV and responds well to the therapeutic interventions with a favorable prognosis in comparison to the HPV-unrelated OPSCC. TNM-8 classifies HPV-related OPSCC and HPV-unrelated OPSCC separately, taking the etiological and prognostic differences of them into consideration. TNM-8 uses $\mathrm{p} 16$ positivity as a surrogate of HPV infection similar to this study. Although some have reported better differentiation of OS according to stages by TNM-8 than by 7th edition TNM classification (7-9), there are also reports indicating that prognoses according to the stages are inadequately differentiated, especially between stage II and III by using TNM-8 for p16positive OPSCC (10-12). Current study showed the stages defined by TNM-8 were inadequate in differentiating OS and DSS. Additionally more than $60 \%$ of HPV-related OPSCC patients were classified into stage I in this study, and further classification of stage I patients should be considered to improve differentiation of prognosis and to allocate equal number of patients to each stage (10).

While HPV-unrelated OPSCC is caused mainly by tobacco smoking and alcoholic consumption, the relationship of HPV-related OPSCC and tobacco smoking has been variously reported $(2,3,13-23)$. Most notably Ang et al. demonstrated that amount of tobacco smoking expressed by PY $>10$ and PY $\leq 10$ had an influence upon OS and progression-free survival (PFS) in HPV-related OPSCC treated by chemoradiation and they proposed a risk classification of OPSCC according to p16 status, smoking habit, and tumor and nodal stages $(2,12)$. Although many studies have shown a statistically significant influence of tobacco smoking on OS $(4,17,19,22,23)$, controversial results have been reported concerning the effect of tobacco smoking on DSS and locoregional control (15) (4) (21). While most studies have studied the patients with HPV-related OPSCC treated by radiotherapy with or without chemotherapy $(16,22,23)$, there is a report refusing the influence of tobacco smoking on OS and DSS in the patients with HPV-related OPSCC treated by transoral robotic surgery (13). In contrary, the study from Canada, where the patients undergoing surgical treatment as well as radiation therapy were reported together, revealed that tobacco smoking has more unfavorable effect upon PFS in the patients treated surgically than in the patients treated by radiation therapy (21). Therefore, the impact of tobacco smoking might be different in various treatment modalities.

This study demonstrated that the patients managed by the treatment including radiation therapy showed no statistically significant difference in DSS by smoking represented by $\mathrm{PY}>30$ or $\mathrm{PY}<30$. In contrast, the patients treated by chemotherapy and surgery showed statistically significant differences in DSS by smoking. Also in Cox multivariate analysis employing smoking and the presence of secondary cancer as covariates, smoking lost a statistically significant impact in DSS in the patients treated by radiation therapy. Radiation therapy seems to reduce the influence of tobacco smoking, although the impact of tobacco smoking continues to exist in the patients undergoing surgery. These findings suggests that the influence of tobacco smoking upon DSS seems to be different according to therapeutic strategy.

In this study, tobacco smoking represented by PY $\geq 30$ vs. $<30$ was used to show the influence of tobacco smoking upon prognosis. We also analyzed the prognosis of never-smokers and searched for multiple dichotomized points of PY and dichotomy at PY $=30$ was found to influence both OS and DSS 
of HPV-related OPSCC with the smallest p-values. Also time interval between cessation of smoking and treatment initiation of OPSCC was examined, but no statistically significant influence upon prognosis was observed.

As a retrospective study, this study has some limitations. Deviation of prognostic factor like the presence of secondary cancer was seen between the patients with $P Y \geq 30$ and $<30$. Secondary cancers were more frequently seen in the patients with $P Y \geq 30$. However, multivariate analysis revealed the presence of secondary cancer lost a statistical significance in DSS. Additionally because of the favorable prognosis of HPV-related OPSCC total number of events in DSS is only 10 and it was difficult to show statistical significances in some analyses. In future, the prospective trial is necessary to elucidate the different impact of smoking upon various treatment modalities. Because many different metrics of tobacco smoking, such as number of PY, never vs. ever smokers, current vs. former smokers, and cessation length of tobacco smoking, were used, common language of expression of tobacco smoking should be defined beforehand (20).

\section{Conclusions}

In this study, TNM-8 stage classification was shown to be inadequate to differentiate prognosis of the patients with HPV-related OPSCC. The amount of tobacco smoking had a statistically significant influence upon OS and DSS in the whole patients, but difference of DSS by the amount of tobacco smoking was not statistically significant in the patients treated by radiation therapy. The effect of tobacco smoking might differ according to the therapeutic modalities.

\section{Abbreviations}

HPV: human papilloma virus, OPSCC: oropharyngeal squamous cell carcinoma, OS: overall survival, DSS: disease-specific survival, TNM: tumor, node, and metastasis, PY: pack year, MRI: magnetic resonance imaging, PET: positron emission tomography, IMRT: intensity modulated radiation therapy, CDDP: cisdiamine-dichloro-platinum.

\section{Declarations}

- Ethical Approval and Consent to participate: This study was approved by the Institutional Review Board (IRB) of National Cancer Center as No. 2017-091 and No. 2018-179, and due to the retrospective nature of the study consent of patient to participate in the study was approved to be waiver.

- Consent for publication: Not applicable in this manuscript

- Availability of supporting data: Data are available from the authors upon reasonable request after permission of the Institutional Review Board of National Cancer Center.

- Competing interests: Following potential competing interests were reported. 
JI reports grants and from ITOCHU and Elekta, personal fees from Hekabio and AlphaTau, and travel reimbursement from Kay's Japan.

KK reports no conflict of interest.

TM reports no conflict of interest.

$\mathrm{YH}$ reports no conflict of interest.

YK reports no conflict of interest.

NM reports no conflict of interest.

GO reports no conflict of interest.

YN reports personal fee from AstraZeneca.

HI reports a research grant from Hekabio, honoraria for lectures from Itochu and AstraZeneca, and personal fee from Hekabio.

KO reports no conflict of interest.

$\mathrm{KI}$ reports no conflict of interest.

$\mathrm{KT}$ reports no conflict of interest.

TKas reports no conflict of interest.

TKan reports no conflict of interest.

SS reports no conflict of interest.

AT reports no conflict of interest.

YS reports no conflict of interest.

FM reports no conflict of interest.

SY reports no conflict of interest.

- Funding: This study is partially financially supported by research grant from ITOCHU. The funding body has nothing to do with content of the study.

- Authors' contributions: JI, KK, NM, GO, FM, and SY designed the study, TM did the p16 immunohistochemistry and diagnosis, $\mathrm{YH}$ reviewed chemotherapy, $\mathrm{YK}$ reviewed radiological examinations, YN, HI, KO, KI, KT, TKas, TKan, SS, AT, YS collected clinical records, the results were 
interpreted by JI, KK, FM, and SY. All the coauthors approved to submit this study to "Radiation Oncology".

- Acknowledgements: not applicable in this manuscript

\section{References}

1. O'Sullivan B, Huang SH, Perez-Ordonez B, et al. Outcomes of hpv-related oropharyngeal cancer patients treated by radiotherapy alone using altered fractionation. Radiother Oncol 2012;103:49-56.

2. K. Kian Ang MD, Ph.D., Jonathan Harris, M.S., Richard Wheeler, M.D.,, Randal Weber MD, David I. Rosenthal, M.D., Phuc Felix Nguyen-Tân, M.D., William H. Westra, M.D., Christine H. Chung, M.D.,, Richard C. Jordan DDS, Ph.D., Charles Lu, M.D., Harold Kim, M.D., Rita Axelrod, M.D., C. Craig Silverman, M.D., Kevin P. Redmond, M.D., and Maura L. Gillison, M.D., Ph.D. Human papillomavirus and survival of patients with oropharyngeal cancer. N Engl J Med 2010;363:24-35.

3. Gillison ML, Zhang Q, Jordan R, et al. Tobacco smoking and increased risk of death and progression for patients with p16-positive and p16-negative oropharyngeal cancer. J Clin Oncol 2012;30:2102-11.

4. Hong AM, Martin A, Chatfield M, et al. Human papillomavirus, smoking status and outcomes in tonsillar squamous cell carcinoma. Int J Cancer 2013;132:2748-54.

5. Lewis JS, Jr., Beadle B, Bishop JA, et al. Human papillomavirus testing in head and neck carcinomas: Guideline from the college of american pathologists. Arch Pathol Lab Med 2018;142:559-597.

6. Rahimi S. Hpv-related squamous cell carcinoma of oropharynx: A review. J Clin Pathol 2020.

7. Husain ZA, Chen T, Corso CD, et al. A comparison of prognostic ability of staging systems for human papillomavirus-related oropharyngeal squamous cell carcinoma. JAMA Oncol 2017;3:358-365.

8. O'Sullivan B, Huang SH, Su J, et al. Development and validation of a staging system for hpv-related oropharyngeal cancer by the international collaboration on oropharyngeal cancer network for staging (icon-s): A multicentre cohort study. Lancet Oncol 2016;17:440-451.

9. Saito $\mathrm{Y}$, Hayashi R, lida $\mathrm{Y}$, et al. Optimization of therapeutic strategy for p16-positive oropharyngeal squamous cell carcinoma: Multi-institutional observational study based on the national head and neck cancer registry of japan. Cancer 2020.

10. De Felice F, Bird T, Michaelidou A, et al. Radical (chemo)radiotherapy in oropharyngeal squamous cell carcinoma: Comparison of tnm 7th and 8th staging systems. Radiother Oncol 2020;145:146-153.

11. Bradish T, Fisher H, Paleri $\mathrm{V}$, et al. How applicable is the tnm 8 staging for human papillomavirus (hpv) related oropharyngeal squamous cell carcinoma (opscc) to a uk population of 106 patients? : A cohort comparison of the tnm 7 and tnm8 staging systems for hpv positive oropharyngeal cancer in a uk population. Eur Arch Otorhinolaryngol 2020.

12. Fakhry C, Zhang Q, Gillison ML, et al. Validation of nrg oncology/rtog-0129 risk groups for hpvpositive and hpv-negative oropharyngeal squamous cell cancer: Implications for risk-based therapeutic intensity trials. Cancer 2019;125:2027-2038. 
13. Roden DF, Hobelmann K, Vimawala S, et al. Evaluating the impact of smoking on disease-specific survival outcomes in patients with human papillomavirus-associated oropharyngeal cancer treated with transoral robotic surgery. Cancer 2020;126:1873-1887.

14. PKS Chan * JC, AC Vlantis, TL Chow, SC Fung, CH Lau, FYH Ng, CS Wong. Smoking, human papillomavirus infection, and p53 mutation as risk factors in oropharyngeal cancer: A case-control study. Hong Kong Medical Journal 2017;23:12-16.

15. Maxwell JH, Kumar B, Feng FY, et al. Tobacco use in human papillomavirus-positive advanced oropharynx cancer patients related to increased risk of distant metastases and tumor recurrence. Clin Cancer Res 2010;16:1226-35.

16. Lassen P, Lacas B, Pignon JP, et al. Prognostic impact of hpv-associated p16-expression and smoking status on outcomes following radiotherapy for oropharyngeal cancer: The march-hpv project. Radiother Oncol 2018;126:107-115.

17. Hawkins PG, Mierzwa ML, Bellile E, et al. Impact of american joint committee on cancer eighth edition clinical stage and smoking history on oncologic outcomes in human papillomavirusassociated oropharyngeal squamous cell carcinoma. Head Neck 2019;41:857-864.

18. Hafkamp HC, Manni JJ, Haesevoets A, et al. Marked differences in survival rate between smokers and nonsmokers with hpv 16-associated tonsillar carcinomas. Int J Cancer 2008;122:2656-64.

19. Chidambaram S, Nakken ER, Kennedy W, et al. Prognostic significance of smoking in human papillomavirus-positive oropharyngeal cancer under american joint committee on cancer eighth edition stage. Laryngoscope 2020;130:1961-1966.

20. Chen SY, Massa S, Mazul AL, et al. The association of smoking and outcomes in hpv-positive oropharyngeal cancer: A systematic review. Am J Otolaryngol 2020;41:102592.

21. Vawda N, Banerjee RN, Debenham BJ. Impact of smoking on outcomes of hpv-related oropharyngeal cancer treated with primary radiation or surgery. Int J Radiat Oncol Biol Phys 2019;103:1125-1131.

22. Platek AJ, Jayaprakash V, Merzianu $M$, et al. Smoking cessation is associated with improved survival in oropharynx cancer treated by chemoradiation. Laryngoscope 2016;126:2733-2738.

23. Mirghani H, Leroy $\mathrm{C}$, Chekourry $\mathrm{Y}$, et al. Smoking impact on hpv driven head and neck cancer's oncological outcomes? Oral Oncol 2018;82:131-137.

\section{Tables}


Table 1. Clinical characteristics and treatments of 142 patients with HPV-related OPSCC.

\begin{tabular}{|c|c|c|c|c|}
\hline & & $\mathrm{n}(\%)$ & & \\
\hline & all & $<$ PY30 & $\geq$ PY30 & $\mathbf{p}$ \\
\hline & $142(100 \%)$ & $98(68.8 \%)$ & $44(31.2 \%)$ & \\
\hline Median age (range) & 63.8 years $(35-84)$ & 62.5 years $(35-84)$ & 65.8 years $(50-82)$ & 0.142 \\
\hline \multicolumn{5}{|l|}{ Gender } \\
\hline male & $118(83.1 \%)$ & $78(79.6 \%)$ & $40(90.9 \%)$ & 0.096 \\
\hline female & $24(16.9 \%)$ & $20(20.4 \%)$ & $4(9.1 \%)$ & \\
\hline \multicolumn{5}{|l|}{ Smoking habit } \\
\hline median PY (range) & 17.3 PY (0-152) & & & \\
\hline No. of non-smoker & $41(28.9 \%)$ & & & \\
\hline $\begin{array}{l}\text { Ex-smoking less than one } \\
\text { year }\end{array}$ & $30(21.1 \%)$ & & & \\
\hline \multirow{2}{*}{\multicolumn{5}{|c|}{ Synchronous and metachronous cancers }} \\
\hline & & & & \\
\hline $\begin{array}{l}\text { yes } \\
\text { no }\end{array}$ & $97(68.3 \%)$ & $74(75.5 \%)$ & $\begin{array}{l}21(47.7 \%) \\
23(52.3 \%)\end{array}$ & 0.006 \\
\hline \multicolumn{5}{|l|}{ Primary Sites } \\
\hline lateral wall & $108(75.7 \%)$ & $74(75.5 \%)$ & $34(77.3 \%)$ & 0.22 \\
\hline anterior wall & $30(21.5 \%)$ & $23(23.5 \%)$ & $7(15.9 \%)$ & \\
\hline posterior wall & $2(1.4 \%)$ & 1 (1.0\%) & $1(2.3 \%)$ & \\
\hline superior wall & $2(1.4 \%)$ & $0(0 \%)$ & $2(4.5 \%)$ & \\
\hline \multicolumn{5}{|l|}{ T-stage (8th) } \\
\hline Tis & $1(0.7 \%)$ & $0(0 \%)$ & $1(2.3 \%)$ & 0.595 \\
\hline T1 & $20(14.1 \%)$ & $14(14.3 \%)$ & $6(13.6 \%)$ & \\
\hline T2 & $77(54.2 \%)$ & $52(53.0 \%)$ & $25(56.8 \%)$ & \\
\hline T3 & $17(12.0 \%)$ & $13(13.3 \%)$ & $4(9.1 \%)$ & \\
\hline T4 & $27(19.0 \%)$ & 19 (19.4\%) & $8(18.2 \%)$ & \\
\hline \multicolumn{5}{|l|}{ N-stage (8th) } \\
\hline No & $23(16.2 \%)$ & $16(16.3 \%)$ & $7(15.9 \%)$ & 0.825 \\
\hline N1 & $101(71.1 \%)$ & $71(72.5 \%)$ & $30(68.2 \%)$ & \\
\hline N2 & 14 (9.9\%) & $9(9.2 \%)$ & $5(11.4 \%)$ & \\
\hline N3 & $4(2.8 \%)$ & $2(2.0 \%)$ & $2(4.5 \%)$ & \\
\hline \multicolumn{5}{|l|}{ Clinical stage (8th) } \\
\hline 0 & $1(0.7 \%)$ & $0(0 \%)$ & $1(2.3 \%)$ & 0.467 \\
\hline 1 & $87(61.3 \%)$ & $61(62.2 \%)$ & $26(59.1 \%)$ & \\
\hline 2 & 25 (17.6\%) & $18(18.4 \%)$ & $7(15.9 \%)$ & \\
\hline 3 & $29(20.4 \%)$ & 19 (19.4\%) & $10(22.7 \%)$ & \\
\hline \multicolumn{5}{|l|}{ Treatment } \\
\hline $\mathrm{OP}$ & $42(29.6 \%)$ & $35(35.6 \%)$ & $7(15.9 \%)$ & 0.084 \\
\hline OP+PORT & $23(16.2 \%)$ & $14(14.3 \%)$ & $9(20.5 \%)$ & \\
\hline OP+POCCRT & $15(10.6 \%)$ & $8(8.2 \%)$ & $7(15.9 \%)$ & \\
\hline RT & $15(10.6 \%)$ & $8(8.2 \%)$ & $7(15.9 \%)$ & \\
\hline CCRT & $47(33.1 \%)$ & $33(33.7 \%)$ & $14(31.8 \%)$ & \\
\hline
\end{tabular}

CCRT: concurrent chemoradiotherapy. OP: operation, POCCRT: postoperative concurrent chemoradiotherapy, PORT: postoperative radiation therapy, PY: pack year, RT: radiation therapy 
Table 2. Multivariate analyses using tobacco smoking and presence of secondary cancer as categorical covariates in various treatment modalities

\begin{tabular}{|c|c|c|c|c|c|c|c|c|c|c|c|c|c|c|c|c|}
\hline & \multicolumn{8}{|c|}{ Overall Survival } & \multicolumn{8}{|c|}{ Disease Specific Survival } \\
\hline & \multicolumn{2}{|c|}{ All patients } & \multicolumn{2}{|c|}{ Patients treated by RT } & \multicolumn{2}{|c|}{ Patients treated by $O P$} & \multicolumn{2}{|c|}{ Patients treated by CT } & \multicolumn{2}{|c|}{ All patients } & \multicolumn{2}{|c|}{ Patients treated by RT } & \multicolumn{2}{|c|}{ Patients treated by OP } & \multicolumn{2}{|c|}{ Patients treated by CT } \\
\hline & $\mathrm{HR}$ & $\mathbf{p}$ & $\mathrm{HR}$ & p & HR & p & $\mathrm{HR}$ & p & HR & p & HR & p & HR & p & HR & p \\
\hline$P Y \geq 30$ vs. $<30$ & 2.78 & $0.029^{*}$ & & 0.15 & & 0.193 & & 0.057 & 4.517 & $0.033^{*}$ & & 0.255 & 11.304 & $0.030^{*}$ & & 0.339 \\
\hline \multirow[t]{4}{*}{$\begin{array}{l}\text { Presence of secondary } \\
\text { cancer }\end{array}$} & 0.338 & $0.024^{*}$ & & 0.056 & 0.125 & $0.009^{*}$ & & 0.058 & & 0.757 & & 0.905 & & 0.703 & & 0.587 \\
\hline & & & & & & & & & & & & & & & & \\
\hline & \multicolumn{6}{|c|}{ CT: chemotherapy, HR: hazard ratio, OP: operation, PY: pack year } & & & & & & & & & & \\
\hline & \multicolumn{4}{|c|}{ * indicates covariate with a statistical significance. } & & & & & & & & & & & & \\
\hline
\end{tabular}

Figures

Fig. 1.

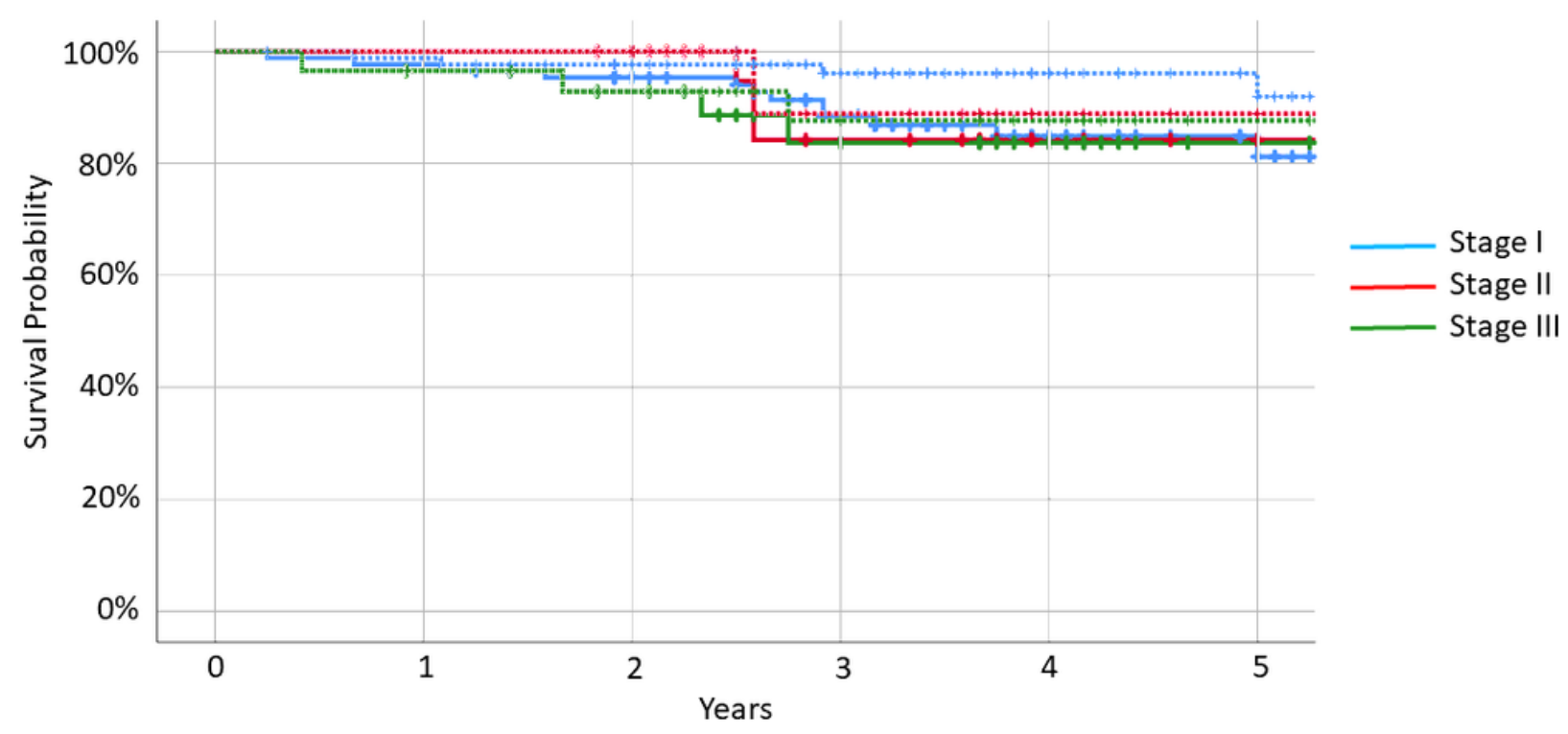

Figure 1

Overall survival (OS, solid line) and disease-specific survival (DSS, broken line) of the 142 patients according to the stage by 8th edition of TNM classification. 
Fig. 2.

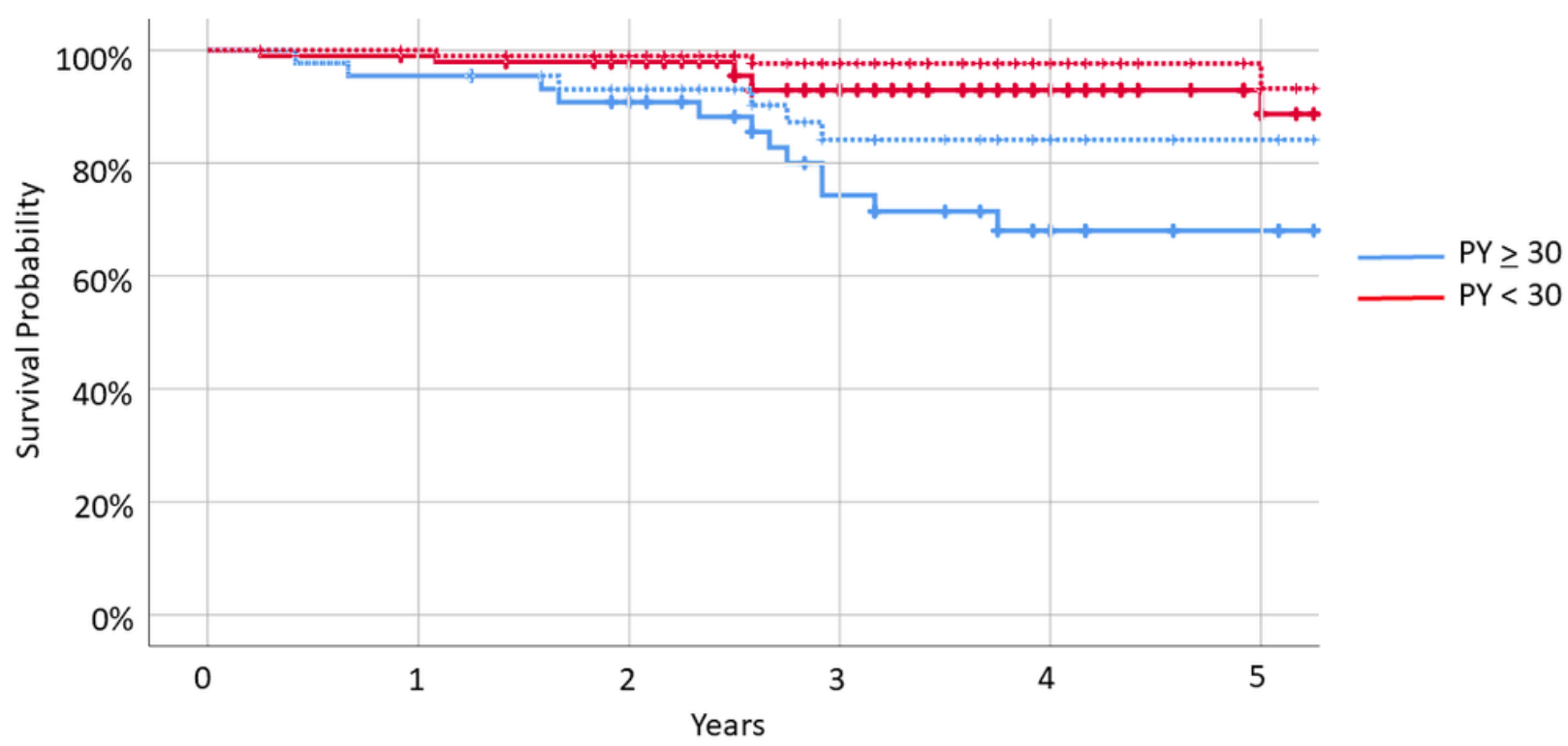

\section{Figure 2}

OS (solid line) and DSS (broken line) of the 142 patients by the amount of tobacco smoking. Between the patients with PY $>30$ and $<30$, there were statistically significant differences in OS $(p=0.003)$ and DSS $(p=0.019)$. 
Fig.3a.

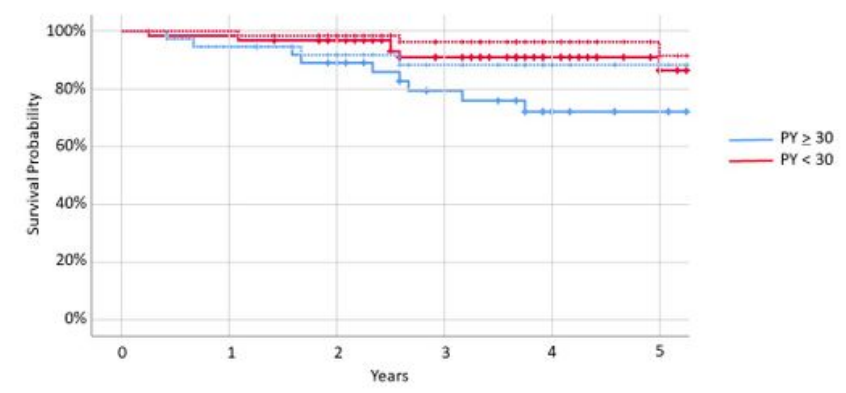

Fig. 3c.

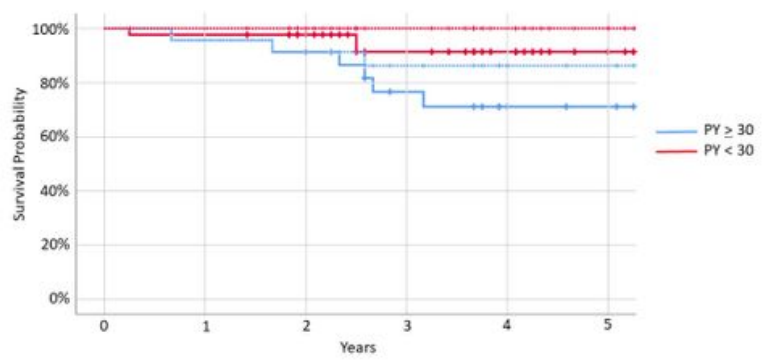

Fig. 3b.

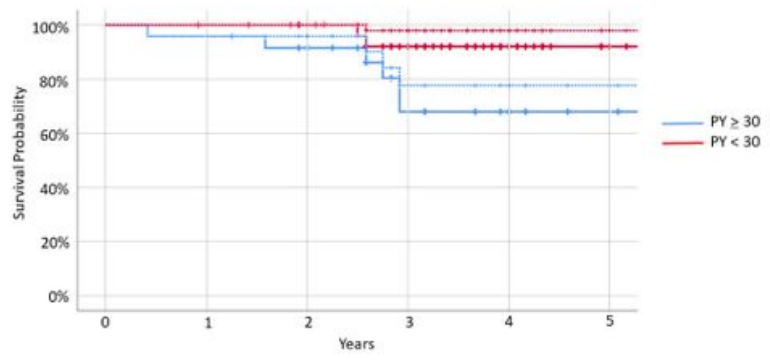

\section{Figure 3}

3a. OS (solid line) and DSS (broken line) by the amount of tobacco smoking in the 100 patients treated by radiation therapy. Between the patients with $\mathrm{PY}>30$ and $<30$, difference in $\mathrm{OS}$ is approaching to a statistical significance $(p=0.084)$, however DSS was not different with a statistical significance $(p=$ 0.137). 3b. OS (solid line) and DSS (broken line) by the amount of tobacco smoking in the 82 patients treated by surgery. Between the patients with $P Y>30$ and $<30$, OS and DSS were both significantly different (OS: $p=0.014$, DSS $p=0.006)$. 3c. OS (solid line) and DSS (broken line) by the amount of tobacco smoking in the 66 patients treated by chemotherapy. Between the patients with $P Y>30$ and $<30$, OS was almost significantly different $(p=0.056)$ and DSS significantly different $(p=0.022)$. 